\title{
Mobilization of xanthine oxidase from the gastrointestinal tract in
} acute pancreatitis

\author{
Susana Granell1, Oriol Bulbena1 ${ }^{1}$ Meritxell Genesca1 ${ }^{1}$ Luis Sabater ${ }^{2}$, \\ Juan Sastre ${ }^{3}$, Emilio Gelpi ${ }^{1}$ and Daniel Closa*1
}

\author{
Address: ${ }^{1}$ Dept. of Experimental Pathology. Institut d'Investigacions Biomèdiques de Barcelona - Consejo Superior de Investigaciones Científicas \\ (IIBB-CSIC), Institut d'Investigacions Biomèdiques August Pi i Sunyer (IDIBAPS), Barcelona, Spain, ²Dept of Surgery, Hospital Clínico \\ Universitario. Valencia, Spain and ${ }^{3}$ Dept. Physiology, Univ. Valencia, Valencia, Spain \\ Email: Susana Granell - sgbbam@iibb.csic.es; Oriol Bulbena - obubam@iibb.csic.es; Meritxell Genesca - mgfbam@iibb.csic.es; \\ Luis Sabater - luis_sabater@hotmail.com; Juan Sastre - Juan.Sastre@uv.es; Emilio Gelpi - egmbam@iibb.csic.es; \\ Daniel Closa* - dcabam@iibb.csic.es \\ * Corresponding author
}

Published: 19 January 2004

BMC Gastroenterology 2004, 4: 1
Received: 24 October 2003

Accepted: 19 January 2004

This article is available from: http://www.biomedcentral.com//47I-230X/4/I

(c) 2004 Granell et al; licensee BioMed Central Ltd. This is an Open Access article: verbatim copying and redistribution of this article are permitted in all media for any purpose, provided this notice is preserved along with the article's original URL.

\begin{abstract}
Background: Xanthine oxidoreductase has been proposed to play a role in the development of local and systemic effects of acute pancreatitis. Under physiologic conditions, the enzyme exists mainly as xanthine dehydrogenase (XDH) but can be converted by proteolytic cleavage to its superoxide-generating form xanthine oxidase (XOD). In addition to its intracellular location XDH/XOD is also associated to the polysaccharide chains of proteoglycans on the external endothelial cell membrane.

In the early stages of acute pancreatitis, this enzyme seems to be arising from its mobilization from the gastrointestinal endothelial cell surface. Taking into account the ability of $\alpha$-amylase to hydrolyze the internal $\alpha-1,4$ linkages of polysaccharides, we wanted to elucidate the involvement of $\alpha$-amylase in $X D H / X O D$ mobilization from the gastrointestinal endothelial cell surface and the relevance of the ascitic fluid (AF) as the source of $\alpha$-amylase in experimental acute pancreatitis.

Methods: Acute pancreatitis was induced in male Wistar rats by intraductal administration of $5 \%$ sodium taurocholate. In another experimental group $3000 \mathrm{U} / \mathrm{Kg} \alpha$-amylase was i.v. administered. The concentrations of XDH, XOD and $\alpha$ amylase in plasma and AF and myeloperoxidase (MPO) in lung have been evaluated. In additional experiments, the effect of peritoneal lavage and the absorption of $\alpha$-amylase present in the AF by an isolated intestine have been determined.
\end{abstract}

Results: Similar increase in XDH+XOD activity in plasma was observed after induction of acute pancreatitis and after i.v. administration of $\alpha$-amylase. Nevertheless, the conversion from XDH to XOD was only observed in the pancreatitis group. Lung inflammation measured as MPO activity was observed only in the pancreatitis group. In addition peritoneal lavage prevented the increase in $\alpha$-amylase and $X D H+X O D$ in plasma after induction of pancreatitis. Finally, it was observed that $\alpha$-amylase is absorbed from the AF by the intestine.

Conclusions: During the early stages of acute pancreatitis, $\alpha$-amylase absorbed from AF through the gastrointestinal tract could interfere with the binding of XDH/XOD attached to glycoproteins of the endothelial cells. Proteolytic enzymes convert XDH into its oxidase form promoting an increase in circulating XOD that has been reported to be one of the mechanisms involved in the triggering of the systemic inflammatory process. 


\section{Background}

Xanthine oxidoreductase has been reported to be one of the most important sources of oxygen free radicals in different pathologies [1]. This enzyme catalyzes the final two steps of the purine degradation pathway: oxidation of hypoxanthine and xanthine to xanthine and uric acid, respectively. Under physiologic conditions, the enzyme exists mainly as xanthine dehydrogenase (XDH), a form that uses $\mathrm{NAD}^{+}$as electron acceptor. Nevertheless, under certain circumstances, XDH can be converted to xanthine oxidase (XOD) by limited proteolytic cleavage. XOD uses molecular oxygen as electron acceptor and consequently generates the superoxide anion [2], a molecule that participates in the generation of other reactive oxygen species including hydrogen peroxide, hydroxyl radical and peroxynitrite.

This enzyme has been proposed to play a role in the development of both local and systemic effects of acute pancreatitis [3-6], an inflammatory process of the pancreatic gland. In experimental models of acute pancreatitis the conversion of XDH to XOD by the action of proteolytic enzymes has been reported [7]. Also, circulating XOD seems to be a source of systemic oxidative stress [6]. This is of importance since the systemic effects of pancreatitis, in particular pulmonary dysfunction characterized by an inflammatory infiltration of the lung parenchyma, are observed in as many as $50 \%$ of patients. Severe forms of pancreatitis progresses in two phases. In the first week the main cause of death is the systemic inflammatory response syndrome while in the second phase, sepsisrelated complications are the main cause of death. Respiratory dysfunction in acute pancreatitis is usually mild and reversible, but in the severe forms of the disease lung injury could be progressive leading to an adult respiratory distress syndrome. This fact appears to be one the most important factors contributing to death in patients with acute pancreatitis during the first week of the disease [8].

In vitro experiments have shown that in addition to its intracellular location $\mathrm{XDH} / \mathrm{XOD}$ is also associated to the external endothelial cell membrane [9]. Other experiments revealed that the addition of heparin inhibits the binding of the enzyme to endothelial cells suggesting that $\mathrm{XDH} / \mathrm{XOD}$ binds to the polysaccharide chains of heparinlike proteoglycans on the endothelial-cell plasma membrane [10]. The binding is apparently of an electrostatic nature: negatively charged groups presumably might interact with some clusters of positively charged amino acid residues of $\mathrm{XDH} / \mathrm{XOD}$ [9]. This is an important fact because during acute pancreatitis circulating XOD seems to be arising from its mobilization from the surface of endothelial cells [11] and not released by the pancreatic tissue. It was noteworthy that during pancreatitis $\mathrm{XDH} /$
XOD is mobilized only from the gastrointestinal system [11].

Amongst the various factors released during acute pancreatitis, $\alpha$-amylase could be suspected to be involved in the mobilization of XDH/XOD attached to glycoproteins of the endothelial cells. This is related to the ability of $\alpha$ amylase to hydrolyze the internal $\alpha-1,4$ linkages of polysaccharides that could affect some glycoproteins present in the extracellular space and disrupt the bindingsite of XDH/XOD. Moreover, in vitro experiments demonstrate that $\alpha$-amylase bind to glycoproteins by carbohydrate-specific interaction, in particular with glycoproteins possessing $\mathrm{N}$-glycans [12]. Enzymatic activity or carbohydrate-specific interaction could explain the displacement of $\mathrm{XDH} / \mathrm{XOD}$ from the glycoprotein binding sites observed during the early stages of acute pancreatitis. On the other hand, transperitoneal absorption from ascitic fluid $(\mathrm{AF})$ is one of the pathways by way of which the products released by damaged pancreas gain access to the systemic circulation. Consequently, the local concentration of $\alpha$-amylase could be greater in the gastrointestinal tract than in the other organs. This could explain the fact that during pancreatitis $\mathrm{XDH} / \mathrm{XOD}$ is mobilized only from the gastrointestinal tract.

Therefore, in the present work, we wanted to elucidate in an experimental model of acute pancreatitis: 1) the involvement of $\alpha$-amylase in $\mathrm{XDH} / \mathrm{XOD}$ mobilization from the gastrointestinal endothelial cell surface and 2) the relevance of the AF as the source of $\alpha$-amylase in this process.

\section{Methods \\ General}

Animals

Male Wistar rats (250-300 g b.w.) were used in all experiments. Animals were housed in a controlled environment and fed with standard laboratory pelleted formula (A04, Panlab, Barcelona, Spain) and tap water ad libitum. This study conformed to European Community (Directive 86/609/EEC) for the use of experimental animals and the institutional committee of animal care and research approved it.

\section{Pancreatitis}

Animals were anesthetized with an i.p. administration of $10 \%$ urethane $(1 \mathrm{ml} / 100 \mathrm{~g}$ b.w.). The biliopancreatic duct was cannulated through the duodenum and the hepatic duct was closed by a small bulldog clamp. Pancreatitis was induced by retrograde perfusion into the biliopancreatic duct of $5 \%$ sodium taurocholate (Sigma, St Louis, Missouri, USA) in a volume of $0.1 \mathrm{ml} / 100 \mathrm{~g}$ b.w. using a perfusion pump (Harvard Instruments, Edenbridge, UK), 0.3 $\mathrm{ml} / \mathrm{min}$ [13]. Control animals received an intraductal 
perfusion of saline solution ( $\mathrm{NaCl} 0.9 \%)$. We have previously reported that, in this model, a significant neutrophil infiltration occurs in the lung $3 \mathrm{~h}$ after the induction of pancreatitis [6]. Therefore this was the time point chosen for all experiments.

\section{Experimental Design}

Effect of $\alpha$-Amylase in XDHIXOD mobilization from endothelial cells In the first experiment we evaluated the ability of $\alpha$-amylase to mobilize $\mathrm{XDH} / \mathrm{XOD}$ from endothelial cells as it occurs during acute pancreatitis. For this purpose we have set up the experimental groups:

- Controls (C): Intraductal infusion of saline solution $(\mathrm{n}=$ 6).

- Experimental acute pancreatitis (EAP): Intraductal infusion of $5 \%$ sodium taurocholate $(n=6)$.

- $\alpha$-Amylase (A): Animals were treated with $3000 \mathrm{U} / \mathrm{kg} \alpha$ amylase (Roche, Basel, Switzerland) given as an intravenous bolus immediately before an intraductal infusion of saline solution. Previously $\alpha$-amylase was purified by Sephadex G-25 chromatography in order to remove ammonium sulfate present in the buffer.

- Heat-denatured $\alpha$-amylase (DA): In order to determine the specificity of $\alpha$-amylase, animals were treated with heat-denatured $\alpha$-amylase before the intraductal perfusion of saline solution $(n=6)$. In this case, the enzyme was previously heated during $60 \mathrm{~min}$ at $100^{\circ} \mathrm{C}$. In these conditions, the residual enzymatic activity observed was less than $1 \%$.

Plasma and lung tissue samples were obtained $3 \mathrm{~h}$ after the intraductal infusion, immediately frozen and maintained at $-80^{\circ} \mathrm{C}$ until $\mathrm{XOD}, \mathrm{XDH}$ and $\alpha$-amylase were measured in plasma and myeloperoxidase (MPO) was measured in lung.

Role of AF and effect of peritoneal lavage in XDHIXOD and $\alpha$ amylase activity in plasma and in lung inflammation

In a second experiment we have evaluated the hypothesis that $\mathrm{AF}$ is the source of $\alpha$-amylase involved in liberating $\mathrm{XDH} / \mathrm{XOD}$ from the gastrointestinal tract. For this purpose, the following experimental groups were established:

- Controls (C): Intraductal infusion of saline solution $(\mathrm{n}=$ 6).

- Experimental acute pancreatitis (EAP): Intraductal infusion of $5 \%$ sodium taurocholate $(n=6)$.

- Experimental acute pancreatitis + peritoneal lavage $(E A P+P L)$ : Intraductal perfusion of $5 \%$ sodium taurocho- late $(n=6)$. In addition, a polyethylene catheter PE-10 (Clay-Adams, UK) was placed into the peritoneal cavity and $60 \mathrm{ml}$ of warm $\left(37^{\circ} \mathrm{C}\right)$ sterile saline solution was infused at a constant flow $(20 \mathrm{ml} / \mathrm{h})$ immediately after induction of pancreatitis.

Plasma samples were obtained $0,0.5,1$ and $3 \mathrm{~h}$ after the intraductal perfusion of taurocholate. AF and lung tissue samples were obtained $3 \mathrm{~h}$ after the induction of pancreatitis, immediately frozen and maintained at $-80^{\circ} \mathrm{C}$

\section{Intestinal absorption of $\alpha$-amylase}

To evaluate the capacity of the intestinal tract on $\alpha$-amylase absorption from the AF we designed an ex vivo experiment incubating an isolated rat intestine with $\mathrm{AF}$ collected from pancreatitis animals $3 \mathrm{~h}$ after the intraductal perfusion of taurocholate. The concentration of amylase released in the vascular perfusate was measured up to $1 \mathrm{~h}$.

Small bowel was isolated as previously described [14]. Briefly, the abdomen was opened and the colon was isolated and removed after ligation of the right and middle colic vessels. The portal vascular pedicle was prepared by transecting all pancreatic, gastric and splenic vessels. The superior mesenteric artery was exposed, cannulated with PE-50 tubing (Clay-Adams, UK) and immediately perfused with $5 \mathrm{ml}$ of saline solution at $4^{\circ} \mathrm{C}$. The portal vein was cannulated with another PE-150 tubing (Clay-Adams, $\mathrm{UK})$, and the small bowel was free from the retroperitoneal tissue. The apertures of the intestinal lumen were closed with a ligature and the small bowel was placed in a recipient containing $25 \mathrm{ml}$ of saline solution at $4{ }^{\circ} \mathrm{C}$ and brought to the perfusion system.

\section{Perfusion system}

Cannulated small bowel was placed into a recipient with $15 \mathrm{ml}$ of saline solution kept at $37^{\circ} \mathrm{C}$. The mesenteric artery was perfused with a modified Krebs-Henseleit buffer containing $200 \mathrm{mg} / \mathrm{dl}$ of glucose at a maximum pressure of $100 \mathrm{~mm} \mathrm{Hg}$. The perfusate was kept at $37^{\circ} \mathrm{C}$ and oxygenated with $95 \% \mathrm{O}_{2}$ and $5 \% \mathrm{CO}_{2}$ during all the perfusion period. The specimen was maintained during $10 \mathrm{~min}$ in these conditions. Then, saline solution was replaced for $15 \mathrm{ml}$ of $\mathrm{AF}$ and the vascular perfusion continued during $60 \mathrm{~min}$. Samples were obtained at 1, 2, 3, 4, $5,10,30$ and $60 \mathrm{~min}$ of the perfusion period.

\section{Biochemical determinations}

$\alpha$-Amylase assay

$\alpha$-Amylase was determined using commercial kits from Sigma, (St Louis, Missouri, USA) according to the supplier's specifications. 
MPO assay

Measured photometrically employing 3,3',5,5'-tetramethylbenzidine as a substrate [15].

$X D H$ and $X O D$ activity

$\mathrm{XOD}$ and $\mathrm{XDH}$ activity were measured fluorometrically at $37^{\circ} \mathrm{C}$ via oxidation of pterin to isoxanthopterin, using methylene blue as an electron acceptor [16].

\section{Statistical analysis}

Data have been expressed as mean \pm SEM. Means of different groups were compared using a one-way analysis of variance. Student's $t$ test was performed for evaluation of
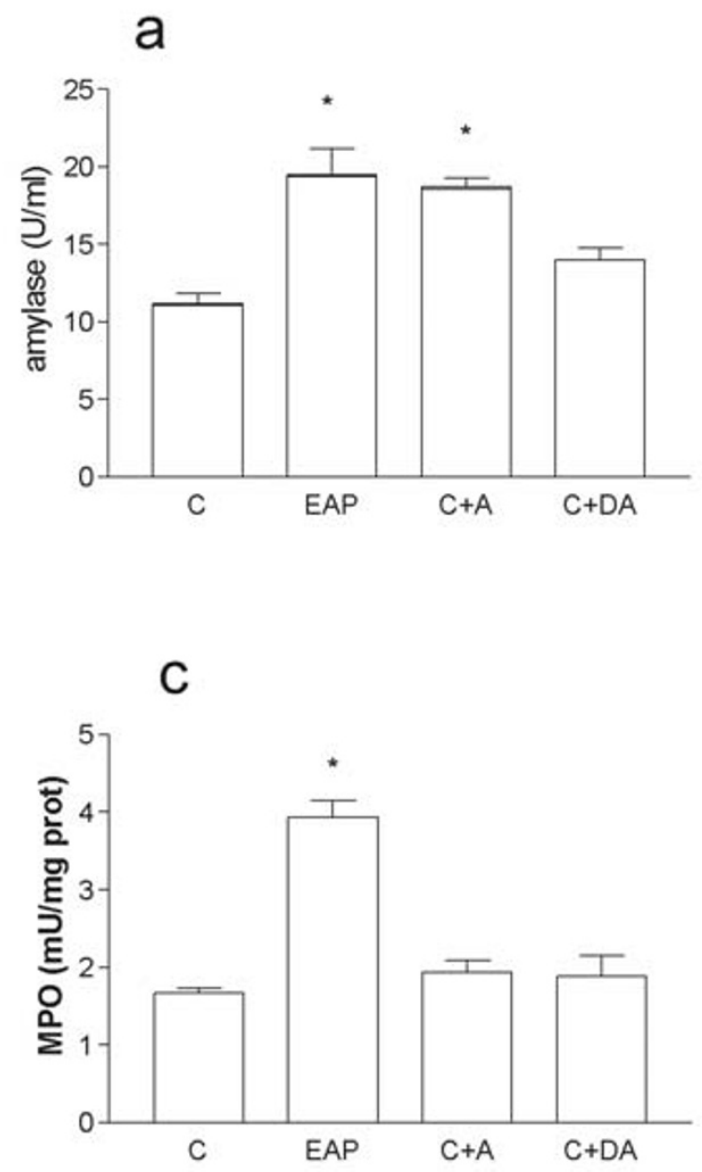

significant differences between groups. Differences were assumed to be significant when $\mathrm{p}<0.05$.

\section{Results \\ Effect of $\alpha$-Amylase in XDHIXOD mobilization from endothelial cells}

Figure 1 shows the effect of acute pancreatitis or $\alpha$-amylase administration on plasma $\alpha$-amylase, $\mathrm{MPO}, \mathrm{XDH}$ and XOD concentration. In our experimental conditions, after $\alpha$-amylase administration the level of XDH+XOD in plasma was similar to that observed during acute pancreatitis (Figure 1a). As expected, denatured $\alpha$-amylasetreated animals showed no significant increase in plasma $\alpha$-amylase activity.
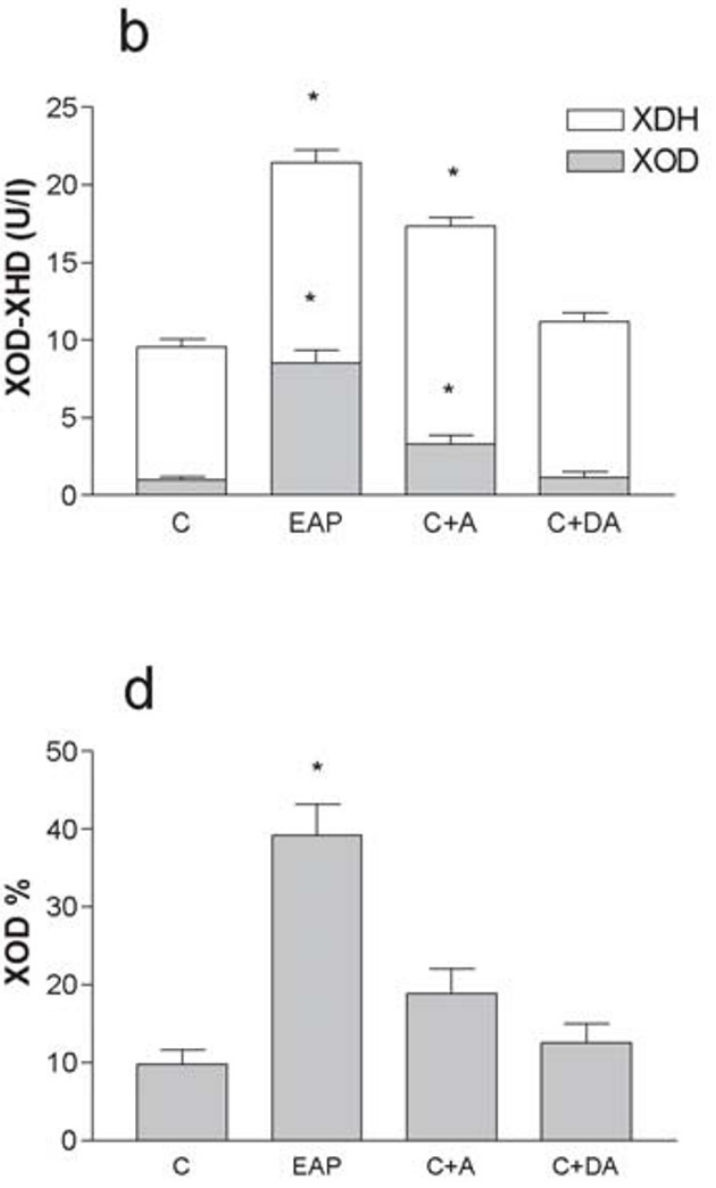

Figure I

Amylase in plasma (a), XDH+XOD in plasma (b), MPO in lung (c) and \% XOD in plasma (d) after induction of EAP or administration of $\alpha$-amylase (A) or heat-denatured $\alpha$-amylase (DA). $\alpha$-Amylase induces an increase in the concentration of $\mathrm{XDH}+\mathrm{XOD}$ similar to that observed during pancreatitis. Nevertheless conversion from XDH to XOD and increased MPO activity in the lung was only observed in the pancreatitis group. $*=p<0.05$ vs control; 
Increased levels of both XDH and XOD activity were observed in pancreatitis group. In the case of $\alpha$-amylase administration a similar increase in XDH activity was observed but, in the case of XOD, the increase was of lesser magnitude than in pancreatitis (Figure 1b). No change in XDH or XOD activity was found when the $\alpha$ amylase administered was heat-denatured. The reduced increase in XOD activity in the $\alpha$-amylase-treated animals is explained by the fact that in the absence of pancreatitis there is no conversion from the dehydrogenase to the oxidase form. This could be clearly observed when the level of XOD was expressed as percentage of the total $\mathrm{XDH}+\mathrm{XOD}$ activity (figure $1 \mathrm{~d}$ ).

$\alpha$-Amylase per se is not enough to induce lung inflammation, as could be observed in figure 1c. Despite obtaining equivalent concentrations of $\alpha$-amylase in plasma after $\alpha$ amylase administration (Figure 1a), neutrophil infiltration, measured as MPO activity was not modified in the lung. Although $\alpha$-amylase induces an increase in the concentration of circulating XDH plus XOD similar to that observed during acute pancreatitis (Figure $1 \mathrm{~b}$ ), the conversion from XDH to XOD was only observed in the pancreatitis group (Figure 1d). In the control group, the percentage of XOD was about $10 \%$ of the total enzyme. This percentage increases to $40 \%$ in pancreatitis, when the proteolytic activity of plasma was higher. In the amylasetreated group, the percentage of XOD was not significantly modified with respect to the control group.

\section{$\mathrm{XDH}+\mathrm{XOD}$ and $\alpha$-amylase in plasma and $\mathrm{AF}$}

Figure 2 shows the kinetics of $\alpha$-amylase and XDH+XOD in plasma, and the final concentration achieved in AF $3 \mathrm{~h}$ after induction of pancreatitis.

As expected, $\alpha$-amylase levels in plasma showed progressive increase after induction of EAP. Total XDH+XOD activity in plasma also increases and achieves significant levels $3 \mathrm{~h}$ after induction of pancreatitis. Finally, the conversion of XDH to XOD also occurs in a time dependent manner during pancreatitis. Interestingly, final $\alpha$-amylase concentration in $\mathrm{AF}$ was over 5 times greater that observed in plasma. In contrast, similar concentrations of XOD and $\mathrm{XDH}$ were observed both in plasma and $\mathrm{AF}$ at the same time point.

\section{Effect of peritoneal lavage in XDHIXOD and $\alpha$-amylase activity in plasma and in lung inflammation}

The increase of total XDH+XOD activity in plasma was prevented when peritoneal lavage was carried out (Figure 3a). Nevertheless, peritoneal lavage did not prevent the $\mathrm{XDH}$ to XOD conversion. Peritoneal lavage also prevented the increase of $\alpha$-amylase observed in plasma after the induction of acute pancreatitis (figure $3 \mathrm{~b}$ ). Finally, the increase in MPO activity in the lung observed after the
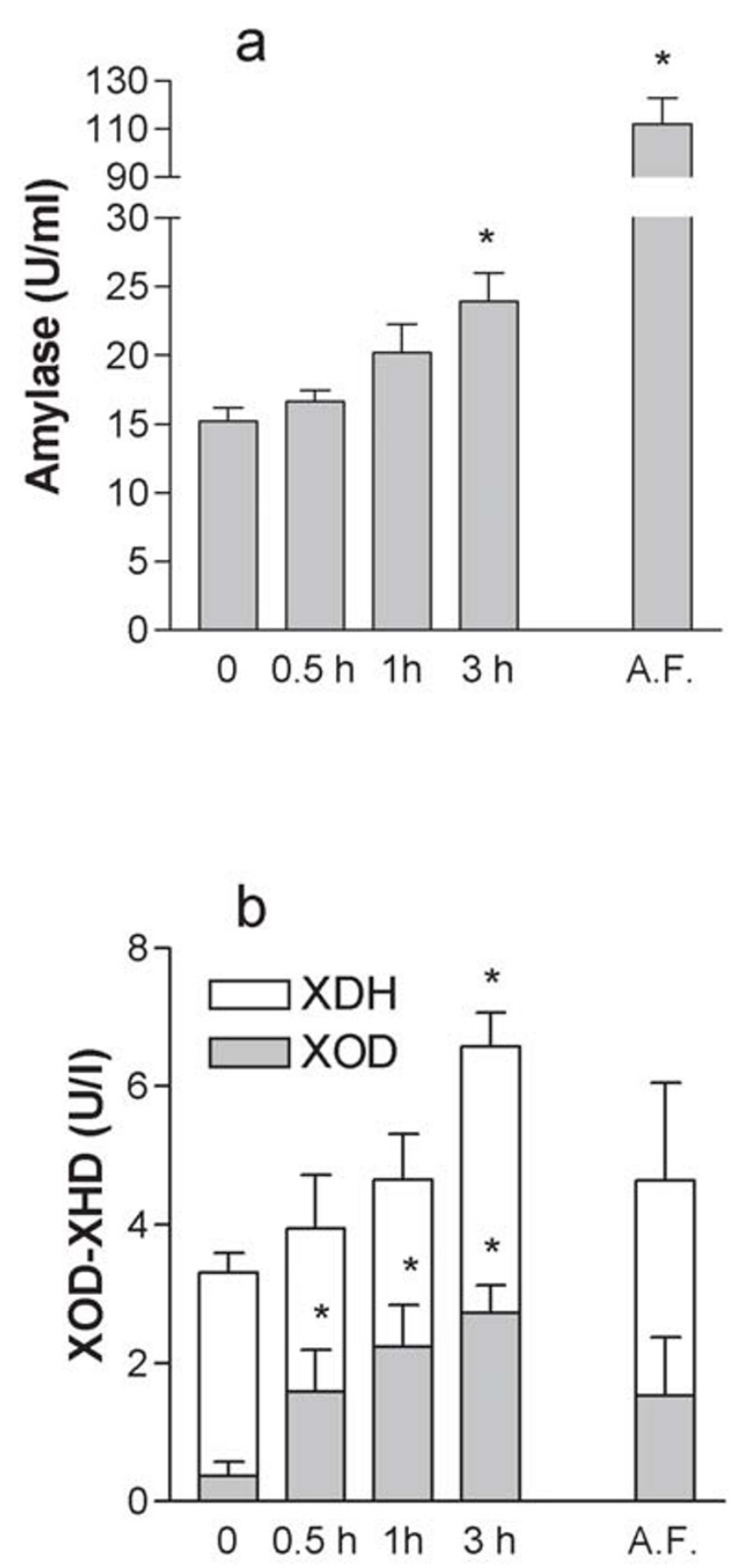

Figure 2

$X O D$ and XDH concentrations (a) and $\alpha$-amylase (b) in plasma and AF after the induction of pancreatitis. Samples of plasma were obtained at $0,0.5, \mathrm{I}$ and $3 \mathrm{~h}$ after induction of pancreatitis. Concentration in AF was evaluated $3 \mathrm{~h}$ after the intraductal infusion of taurocholate. $*=p<0.05$ vs time $=0$.

induction of pancreatitis was also prevented by peritoneal lavage (Figure 3c). 

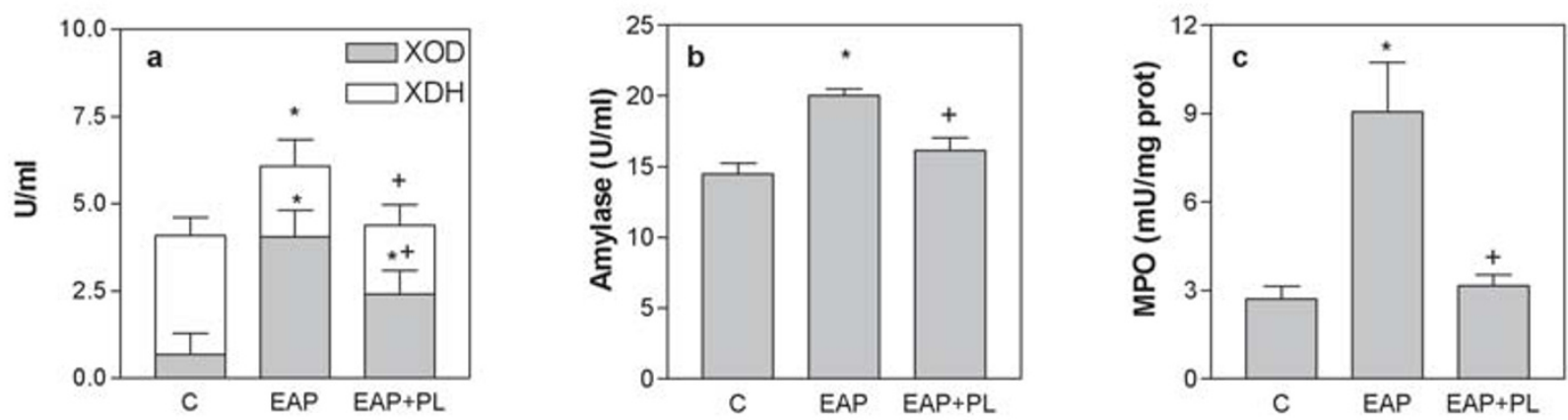

Figure 3

Effect of peritoneal lavage (PL) in XDH+XOD (a) and $\alpha$-amylase (b) plasma levels, and in MPO activity (c) in lung. Peritoneal lavage prevents the increase found in $\alpha$-amylase, MPO and total XDH+XOD, but does not affect the increase in the $\%$ of $X O D$ observed during EAP. $*=p<0.05$ vs control; $+=p<0.05$ vs pancreatitis.

\section{Intestinal absorption of $\alpha$-amylase}

Figure 4 depicts the concentration of $\alpha$-amylase measured in the vascular perfusate at the output of the isolated intestine when incubated in AF. $\alpha$-Amylase was undetectable when the intestine was incubated in saline solution (data not shown). When intestine was incubated in AF $\alpha$-amylase in the vascular perfusate showed a rapid increase, which achieved a maximal concentration in $10 \mathrm{~min}$ and was maintained until the end of the experiment (60 min).

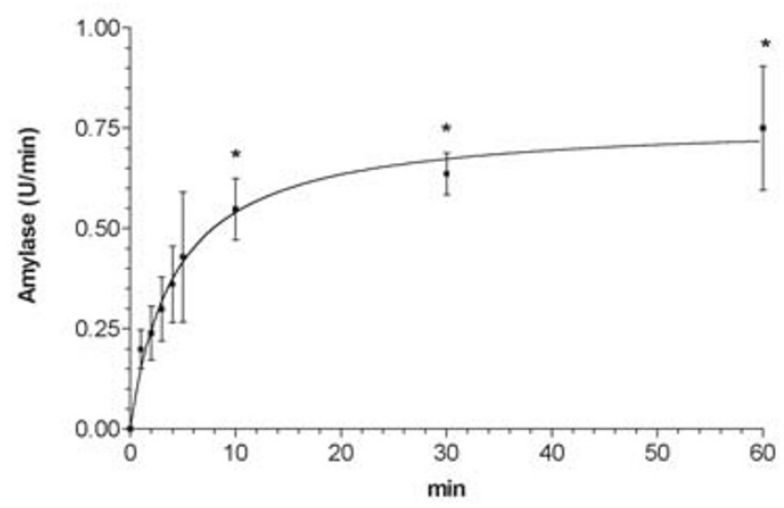

\section{Figure 4}

$\alpha$-Amylase absorption from isolated perfused intestine incubated with AF obtained from animals with pancreatitis. Samples of the perfusion buffer were obtained after placing the organ in the $A F t=0$. Maximal concentration was achieved $10 \mathrm{~min}$ after the initiation of the incubation and maintained for I h. $*=p<0.05$ vs. time $=1$ min.

\section{Discussion}

The involvement of XOD-derived oxygen free radicals in the pathogenesis of acute pancreatitis has been extensively reported [3-5]. In pancreas, conversion of XDH to the free radical-generating form XOD occurs during pancreatitis and is due to the intrapancreatic proteolytic activity. In addition, total $\mathrm{XDH}+\mathrm{XOD}$ activity in plasma increases significantly after the induction of pancreatitis. In the early stages of acute pancreatitis, circulating XOD may act at distant organs generating the superoxide radical species that promote P-selectin expression and recruitment of neutrophils [6]. Pulmonary endothelial cells seem to be particularly sensitive, since systemic XOD-induced Pselectin up-regulation was detected only in the lung [17]. This sensitivity could be also related with the environment generated by the disease, with high concentrations of circulating cytokines, activation of complement system and other potentially deleterious mechanisms operating.

It could be suspected that, as it occurs with other enzymes, the increased XDH+XOD activity observed in plasma during pancreatitis is related with the release of this enzyme from pancreatic tissue. Nevertheless, in physiological conditions, $\mathrm{XDH} / \mathrm{XOD}$ is also bound to the polysaccharide chains of heparin-like proteoglycans existing on the external surface of the endothelial cells $[9,10]$. We have recently reported that during pancreatitis the increase of $\mathrm{XDH} / \mathrm{XOD}$ observed in plasma is related with the mobilization of the XDH/XOD enzyme bound to the surface of the endothelial cells, in particular, from the endothelial cells of the gastrointestinal tract [11].

Serum $\alpha$-amylase is one of the most frequently used tests for the diagnosis of acute pancreatitis [18]. Amongst the 
various factors released during acute pancreatitis, $\alpha$-amylase could be involved in the mobilization of XDH/XOD attached to glycoproteins of the endothelial cells. This is related to the ability of $\alpha$-amylase to hydrolyze the internal $\alpha-1,4$ linkages of polysaccharides that could affect some glycoproteins and disrupt the binding-site of $\mathrm{XDH} /$ XOD. On the other hand, in vitro experiments demonstrated that $\alpha$-amylase binds by carbohydrate-specific interaction to glycoproteins possesing N-glycans [12]. Thus, the enzyme could also displace XDH/XOD through competition for the binding site.

To evaluate this possibility, we administered an amount of $\alpha$-amylase tailored to induce a final concentration in plasma similar to that observed in experimental pancreatitis. In this condition, we observed an increase in $\mathrm{XOD}+\mathrm{XDH}$ concentration in plasma similar to that observed during pancreatitis (Figure 1b).

The effect of $\alpha$-amylase appears to be not due to unspecific interactions since heat-denaturalized $\alpha$-amylase failed to induce the increase in $\mathrm{XDH}+\mathrm{XOD}$ activity in plasma (Figure 1b). Nevertheless, the possibility that heat treatment inhibits the glycoprotein binding site of $\alpha$-amylase cannot be excluded. In fact, there is no evidence in the literature indicating the capability of $\alpha$-amylase to hydrolize membrane bound glycoprotein. On the other hand, it has been reported that $\alpha$-amylase is able to digest proteoglycogen [19], indicating that this enzyme could act on proteinbound polysaccharides.

In addition, $\alpha$-amylase administration had effect only in the mobilization of XDH/XOD but the enzyme remains mainly in its dehydrogenase form. This form did not generate superoxide radicals, probably for this reason we found that, despite the increase in total circulating $\mathrm{XDH}+\mathrm{XOD}$, lung inflammation was observed only when there was a significant increase in the oxidase form of the enzyme (Figures 1c and 1d). The conversion occurs as a consequence of proteolytic cleavage of the enzyme probably due to the activation of proteolytic enzymes and this fact occurs only during pancreatitis. In fact, proteolytic conversion to the oxidase form was only observed after induction of pancreatitis (figure 1).

These results indicate that, effectively, the pancreatic $\alpha$ amylase released during acute pancreatitis interferes with the binding of $\mathrm{XDH} / \mathrm{XOD}$ to endothelial cells, thus contributing to the increase observed in the plasma concentration of this enzyme. The proteolytic activity associated with the disease converts the enzyme to the superoxidegenerating form, contributing to the inflammation in the lung through a free radical-dependent mechanism.
Nevertheless, during pancreatitis, mobilization of XDH/ $\mathrm{XOD}$ is restricted to the gastric and intestinal endothelial surface [11]. To explain this fact we hypothesized that $\alpha$ amylase present in the AF could be important in this regard. This fluid contains biologically active agents, such as pancreatic lipase, phospholipase A2, $\alpha$-amylase, proteases, complement factors, kinins, and inflammatory mediators, which are released into the peritoneal cavity during acute pancreatitis [20]. Products released by pancreas gain access to the systemic circulation by different pathways: transperitoneal absorption from AF, direct transfer into veins draining the pancreas and absorption into pancreatic and retroperitoneal lymphatics [21].

Local concentration of $\alpha$-amylase absorbed from AF could be greater in the gastrointestinal tract than in other organs. When comparing the concentrations observed in plasma and in the AF, we observed that the enzyme concentration is about five fold higher in AF (Figure 2). This fact does not occur for XDH/XOD. Therefore, a high absorption and local concentration of $\alpha$-amylase in the gastrointestinal vessels could be expected and consequently, its effect on the XDH/XOD binding could be most important in this organ.

To verify this possibility we performed a peritoneal lavage in a group of animals, immediately after the induction of pancreatitis (Figure 3). When AF was removed by the lavage, the increase in $\alpha$-amylase activity in plasma was not observed and the inflammatory process in the lung was also prevented. With respect to the $\mathrm{XDH} / \mathrm{XOD}$, there was no increase in total enzyme concentration. This fact agrees with the hypothesis that $\alpha$-amylase absorbed from the AF is needed to mobilize XDH/XOD and that the proteolytic conversion of the enzyme occurs through a different, independent mechanism. On the other hand, peritoneal lavage results in a reduction of XOD levels in plasma, but the difference is not very high. By contrast, differences observed in MPO levels in the lung as a consequence of peritoneal lavage was highly significant. This fact suggests that peritoneal lavage also removes other mediators involved in the development of the lung inflammation and pointed out the importance of the interactions between different pro-inflammatory pathways in this process. This consideration applies also to other mechanisms suggested by these results. For example, the observed conversion from $\mathrm{XDH}$ to XOD occurs by proteolytic cleavage but the particular proteolytic enzymes involved in this conversion remains to be elucidated. In addition, there are considerable interspecific differences in the levels of both proteolytic activity and protease inhibitors. Obviously, further studies are needed in humans to verify the applicability of these results to human disease. 


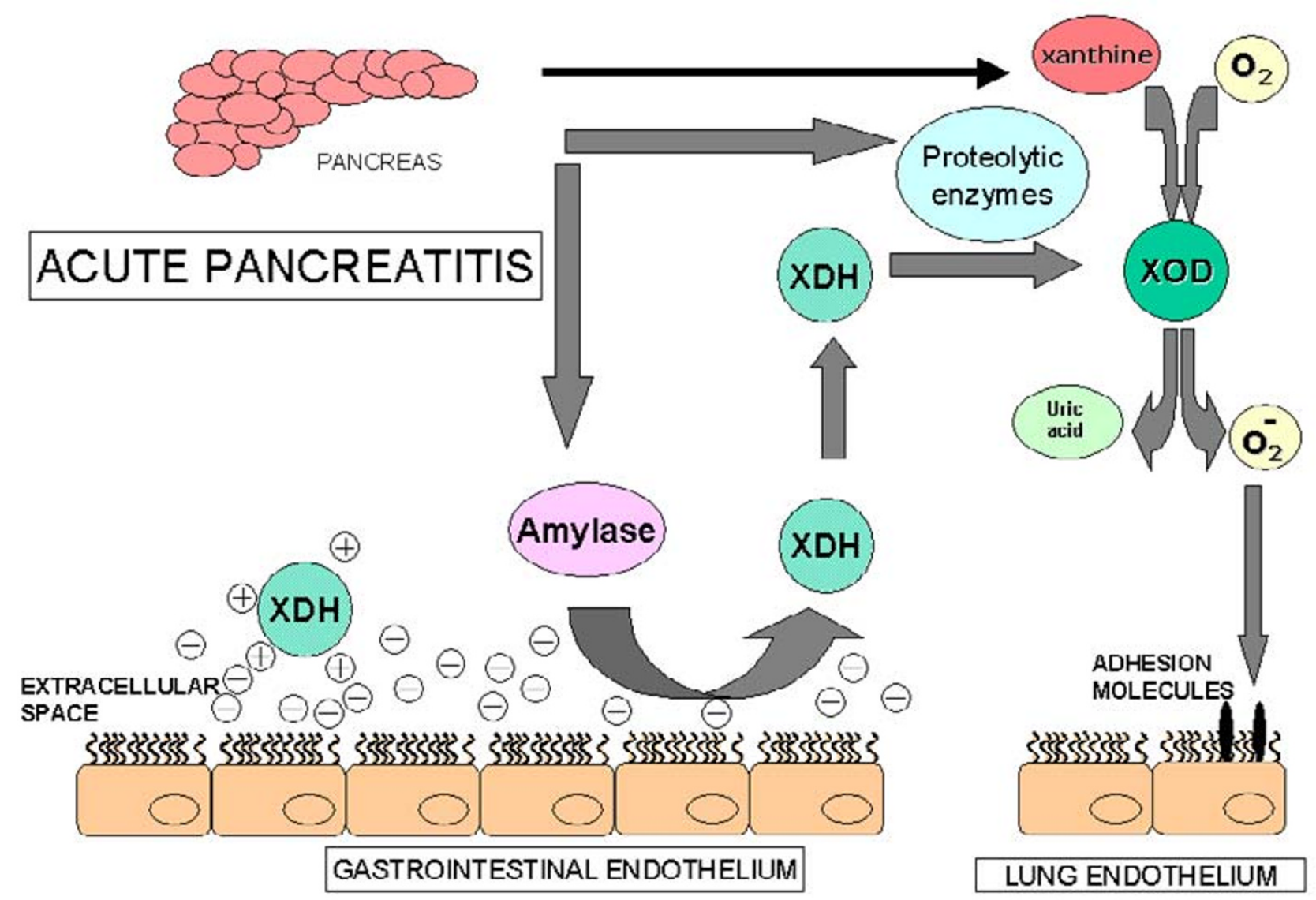

Figure 5

Suggested mechanism of the mobilization of XDH/XOD from the surface of the endothelial cells of gastrointestinal tract during acute pancreatitis: $\alpha$-Amylase released from the pancreas acts on the polysaccharide chains of the endothelial cells and mobilizes the enzyme, mainly present as $\mathrm{XDH}$. Proteolytic enzymes convert this enzyme into its oxidase form. In addition, acinar cells from the damaged pancreas release xanthine resulting from purine catabolism. Then, the simultaneous presence of xanthine and XOD in the bloodstream constitutes a circulating free radical-generating system that could induce the up regulation of cell adhesion molecules and the inflammatory response in the lungs.

The ability of the gastrointestinal tract to absorb $\alpha$-amylase from the AF was also directly assessed by incubating an isolated intestine with AF obtained from animals with pancreatitis and measuring the enzyme concentration in the vascular perfusate (Figure 4). Results indicate that enzyme absorption was fast and that the maximum value was achieved only ten minutes after the incubation. By contrast, $\mathrm{XDH} / \mathrm{XOD}$ activity was not observed in the eluate, discarding a significant intraperitoneal absorption of this enzyme. This fact was not surprising since the concentration of XDH/XOD in AF is not increased with respect to that in plasma (Figure 2).
Peritoneal lavage has been suggested to be of therapeutical value in patients with acute pancreatitis. Some experimental studies reported beneficial effects of this method in reducing the systemic effects of pancreatitis, but in general, experimental work started the lavage immediately after the induction of pancreatitis [22]. In clinical work, results are by far less clear and our results could help to explain this fact [23]. Lavages are performed in patients only after acute pancreatitis is diagnosed. Since the increased $\alpha$-amylase concentration is one of the usual parameters for the diagnosis of acute pancreatitis, in the clinical practice the lavage does not start until the $\alpha$-amy- 
lase concentration increases; too late to prevent the release of XDH/XOD induced by $\alpha$-amylase.

It is important to consider that $\alpha$-amylase could enter the systemic circulation through different pathways. In a previous work, using the same experimental model, we have observed a similar involvement for lymphatic draining and peritoneal absorption pathways [24]. Nevertheless, results obtained in this work suggest that only the $\alpha$-amylase uptake by the splanchnic circulation is involved in the mobilization of $\mathrm{XDH} / \mathrm{XOD}$ from the gastrointestinal endothelium.

In conclusion, our results indicate that during pancreatitis, $\alpha$-amylase absorbed from $\mathrm{AF}$ in the gastrointestinal tract interferes the binding of $\mathrm{XDH} / \mathrm{XOD}$ attached to the glycoproteins of the endothelial cells (Figure 5). The proteolytic activity associated with the disease converts the enzyme in the superoxide-generating form, inducing the inflammation in the lung through a free radical-dependent mechanism.

\section{List of abbreviations}

Ascitic fluid: AF; EAP: Experimental acute pancreatitis; MPO: Myeloperoxidase; PL: Peritoneal lavage; XDH Xanthine Dehydrogenase; XOD: Xanthine Oxidase.

\section{Competing interests}

None declared.

\section{Authors' contributions}

SG carried out the experimental animals models and biochemical determinations. OB participated in the design of the study and performed the statistical analysis. MG carried out the experiments with the isolated intestine. LS and JS participated in the design and coordination of the study. EG drafted the manuscript and DC conceived the study and participate in its design and coordination. All authors read and approved the final manuscript.

\section{Note}

*IIBB-CSIC: Institut d'Investigacions Biomèdiques de Barcelona - Consejo Superior de Investigaciones Científicas; **IDIBAPS: Institut d'Investigacions Biomèdiques August Pi i Sunyer.

\section{Acknowledgements}

This work has been supported by FISss grants 0I/0949 and PI020286; S.Granell and M.Genesca are recipients of IDIBAPS predoctoral grants.

\section{References}

I. Linder N, Rapola J, Raivio KO: Cellular expression of xanthine oxidoreductase protein in normal human tissues. Lab Invest 1999, 79(8):967-974.

2. Parks DA, Granger DN: Xanthine oxidase: Biochemistry, distribution and physiology. Acta Physiol Scand Suppl 1986, 548:87-99.
3. Nonaka A, Manabe T, Tamura K, Asano N, Imanishi K, Tobe T: Changes of xanthine oxidase, lipid peroxidation and superoxide dismutase in mouse acute pancreatitis. Digestion 1989 , 43:4I-46.

4. Nordback IH, Cameron JL: The mechanism of conversion of xanthine dehydrogenase to xanthine oxidase in acute pancreatitis in the canine isolated pancreas preparation. Surgery I991, II 3:90-97.

5. Cassone E, Maneschi EMT, Faccas JG: Effects of allopurinol on ischemic experimental pancreatitis. Int J Pancreatol 1991, 8:227-234.

6. Folch E, Gelpí E, Roselló-Catafau J, Closa D: Free radicals generated by xanthine oxidase mediate pancreatitis-associated organ failure. Dig Dis Sci 1998, 43:2405-24I0.

7. Closa D, Bulbena O, Hotter G, Roselló-Catafau J, Fernández-Cruz L, Gelpí E: Xanthine oxidase activation in cerulein- and taurocholate-induced acute pancreatitis in rats. Arch Int Physiol Bioch Bioph 1994, 102:167-170.

8. Buter A, Imrie CW, Carter CR, Evans S, McKay CJ: Dynamic nature of early organ dysfunction determines outcome in acute pancreatitis. Br J Surg 2002, 89:298-302.

9. Houston M, Estevez A, Chumley P, Aslan M, Marklund S, Parks DA, Freeman BA: Binding of xanthine oxidase to vascular endothelium. J Biol Chem 1999, 274:4985-4994.

10. Adachi T, Fukushima T, Usami Y, Hirano K: Binding of human xanthine oxidase to sulphated glycosaminoglycans on the endothelial-cell surface. Biochem J 1993, 289:523-527.

II. Granell S, Gironella M, Bulbena O, Panes J, Mauri M, Sabater L, Aparisi L, Gelpi E, Closa D: Heparin mobilizes xanthine oxidase and induces lung inflammation in acute pancreatitis. Crit Care Med 2003, 31:525-530.

12. Matsushita H, Takenaka M, Ogawa H: Porcine Pancreatic $\alpha$-amylase Shows Binding Activity toward N-Linked Oligosaccharides of Glycoproteins. J Biol Chem 2000, 277:4680-4686.

13. Aho HJ, Suonpää K, Ahola RA, Nevalainen TJ: Experimental pancreatitis in the rat.-Ductal factors in sodium taurocholateinduced acute pancreatitis. Exp Path 1984, 25:73-79.

14. Genesca M, Sola A, Miquel R, Pi F, Xaus C, Alfaro V, Hotter G: Role of changes in tissular Nucleotides on the development of apoptosis during ischemia/reperfusion in rat small bowel. $\mathrm{Am}$ J Patho 2002, 161:1839-1847.

15. Trush MA, Egner PA, Kensler TW: Myeloperoxidase as a biomarker of skin irritation and inflammation. Fd Chem Toxic 1994, 32:143-147.

16. Beckman JS, Parks DA, Pearson JD, Marshall PA, Freeman BA: A sensitive fluorometric assay for measuring xanthine dehydrogenase and oxidase in tissues. Free Radic Biol Med 1989, 6:607-615.

17. Folch E, Salas A, Panés J, Gelpí E, Roselló-Catafau J, Anderson DC, Navarro S, Piqué JM, Fernández-Cruz L, Closa D: Role of P-selectin and ICAM-I in pancreatitis-induced lung inflammation in rats: Significance of oxidative stress. Ann Surg 1999, 230:792-799.

18. Smotkin J, Tenner S: Laboratory diagnostic test in acute pancreatitis. J Clin Gastroenterol 2002, 34:459-462.

19. Romero JM, Curtino JA: C-chain-bound glycogenin is released from proteoglycogen by isoamylase and is able to autoglucosylate. Biochem Biophys Res Commun 2003, 305:8I I-8I4.

20. Svensson C, Sjodahl R, Lilja I, Ihse I: The role of ascites and phospholipase $A_{2}$ on peritoneal permeability changes in acute experimental pancreatitis. Int J Pancreatol 1990, 6:71-79.

21. Dugernier T, Laterre PF, Reynaert MS: Ascites fluid in severe acute pancreatitis: from pathophysiology to therapy. Acta Gastroenterol Belg 2000, 63(3):264-268.

22. Dugernier T, Reynaert MS, Deby-Dupont G, Roeseler JJ, Carlier M, Squifflet JP, Deby C, Pincemail J, Lamy M, De Maeght S: Prospective evaluation of thoracic-duct drainage in the treatment of respiratory failure complicating severe acute pancreatitis. Intensive Care Med 1989, 15(6):372-378.

23. Niederau C, Crass RA, Silver G, Ferrell LD, Grendell JH: Therapeutic regimens in acute experimental hemorrhagic pancreatitis. Gastroenterology 1988, 95:1648-1657.

24. Folch E, Closa D, Gelpí E, Roselló-Catafau J: Effect of peritoneal lavage and lymph ligature on systemic complications of experimental acute pancreatitis. Dig Dis Sci 2000, 45(5):909-914. 


\section{Pre-publication history}

The pre-publication history for this paper can be accessed here:

http://www.biomedcentral.com/1471-230X/4/1/prepub

Publish with Bio Med Central and every scientist can read your work free of charge

"BioMed Central will be the most significant development for disseminating the results of biomedical research in our lifetime. " Sir Paul Nurse, Cancer Research UK

Your research papers will be:

- available free of charge to the entire biomedical community

- peer reviewed and published immediately upon acceptance

- cited in PubMed and archived on PubMed Central

- yours - you keep the copyright
BioMedcentral 